ARCHIVO ESPAÑOL DE ARTE, LXXXI, 323

JULIO-SEPTIEMBRE 2008, pp. 315-322

ISSN: 0004-0428

\title{
UN ORIGINAL DESCONOCIDO (O PERDIDO) Y TRES VERSIONES DEL RETRATO DEL CARDENAL SOLÍS
}

Del retrato del Cardenal Solís se conocían dos versiones o más propiamente copias, si entendemos copia como la reproducción fiel o imitación de una obra con las técnicas y procedimientos ordinarios, del pintor del XVIII Sevillano José Rubira (Sevilla 1747 -Guadix 1787) ${ }^{1}$ una que se encuentra en el Monasterio de Santa Paula y otra en el Palacio Arzobispal de Sevilla.

De José Rubira, hijo del también pintor Andrés Rubira del que fue alumno del cual quedó huérfano cuando tenía 13 años de edad ${ }^{2}$, sabemos que se dedicó especialmente al retrato ejecutando también obras de pequeño tamaño al óleo, temple y pastel, dedicándose también a la miniatura y ocasionalmente a la escultura. También se sabe que debido a la carencia de encargos artísticos en esta época en la diócesis sevillana, se tuvo que dedicar para subsistir a otros menesteres como trabajos vinculados a una fábrica de carruajes. De los pocos datos que tenemos de él sabemos es un pintor que trabaja en la segunda mitad del XVIII, que trabajó al servicio del Cardenal Solís y que según parece, éste quiso llevarle a Roma en 1769, cuando asistió al cónclave que tuvo lugar a la muerte del papa Clemente VIII. Sin embargo Rubira no llegó a realizar este viaje, no sabemos por qué motivos, con lo cual perdió una importante ocasión de contemplar el ambiente artístico romano y así aumentar su formación artística. Sabemos también que fundó junto a Blas Molina y Luis Pérez una academia particular ${ }^{3}$, experiencia que sirvió como circunstancia preparatoria de para la creación posterior de la Escuela de Tres Nobles artes de Sevilla. También sabemos que en 1783 ganó el primer premio de un concurso que convocó dicha escuela y que es uno de los escasos testimonios de su producción que conocemos en el presente, se trata de una copia de La sagrada Familia de Murillo y que actualmente se conserva en la National Gallery de Londres, en donde se observa que tenía unas grandes dotes para la copia.

De los retratos anteriormente mencionados que realiza Rubira también como copias o versiones, el artista aparte de consignar su firma, indica que son un copias de un original que poseyó el Marqués de Loreto en Sevilla ${ }^{4}$, según lo cita el Conde de Maule y que era obra del pintor Italiano Pompeo Batoni firmado y fechado en Roma en 1769. Estas copias debieron ejecutarse hacia 1774, pues ésta es la fecha que aparece en el retrato del Cardenal que pertenece al Monasterio de Santa Paula de Sevilla (con la inscripción en el brazo del sillón "J.Rb. ${ }^{\circ}$ año 1774").

El retratado Francisco Solís Folch de Cardona nació en Madrid en 1713. Existen dudas sobre el lugar exacto de nacimiento del cardenal Solís: las distintas fuentes consultadas lo hacen variar entre 1705 en Salamanca o el 16 de febrero de 1713 en Madrid. Hijo de José de Solís y Gante,

\footnotetext{
1 VAldivieso GonZÁlez, E., Historia de la Pintura Sevillana, siglos XIII al XX, Focus, Ediciones Guadalquivir, Sevilla, 1986, p. 577.

2 Cean Bermúdez, J. A., Diccionario de artistas, Madrid, 1802, sección VA, p. 147.

3 Valdivieso González, E., Historia de la Pintura Sevillana, siglos XIII al XX, Focus, Ediciones Guadalquivir, Sevilla, 1986, p. 577.

${ }^{4}$ Urrea Fernández, Jesús, La Pintura Italiana del Siglo XVIII en España, Publicaciones del Departamento de Historia del Arte, Valladolid, 1977, p. 247.
} 


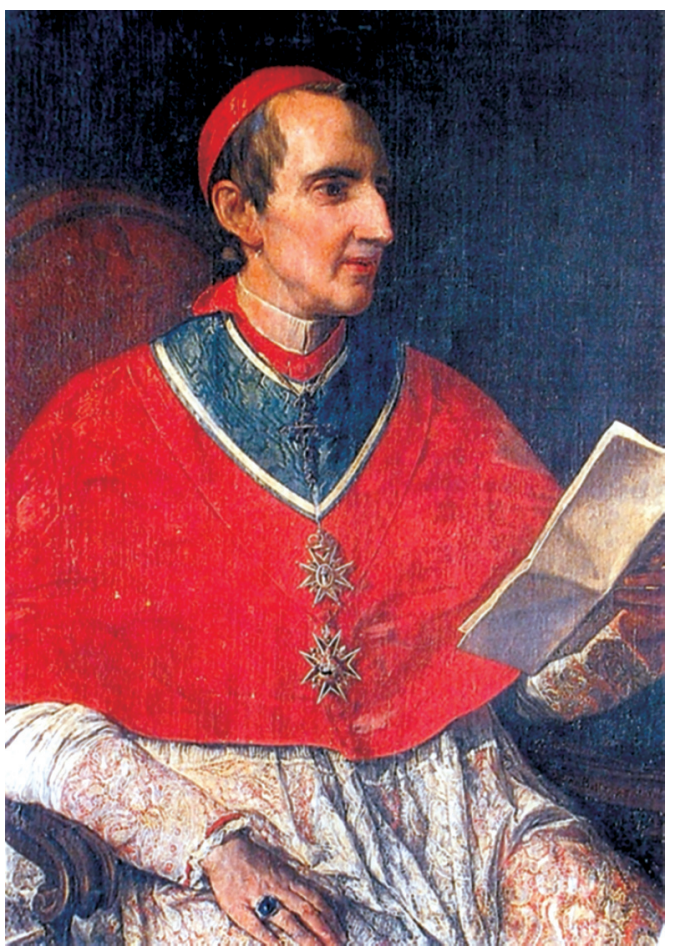

Fig. 1. José Rubira. Cardenal Solís. Sevilla. Monasterio de Santa Paula.

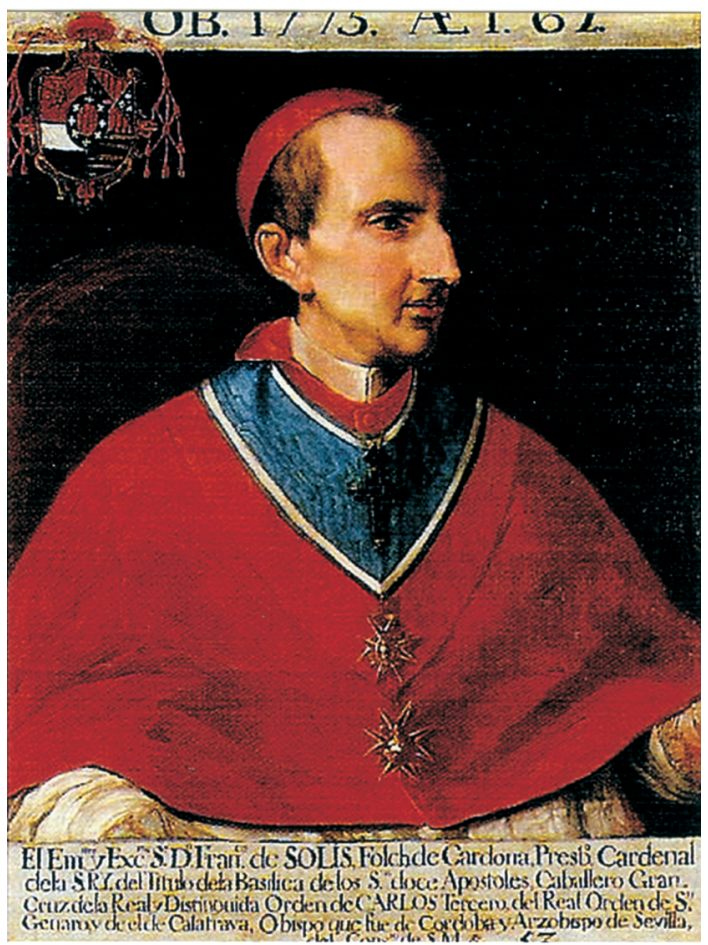

Fig. 2. José Rubira (?). Cardenal Solís. Sevilla. Palacio Arzobispal.

marqués de Castelnuovo, conde de Saldueña y tercer duque de Montellano y uno de los miembros fundadores de la Real Academia Española (fundada en 1713), grande de España, caballero de la orden de Santiago, mayordomo y caballerizo mayor del Cardenal infante y de Josefa Folch de Cardona, marquesa de Castelnovo y de Pons. Su hermano, José Solís Folch de Cardona, fue el tercer virrey de Nueva Granada (actual Colombia) y más tarde ingresó en la orden Franciscana. Estudió en la Universidad de Alcalá. Fue sumiller de Cortina de Felipe V, canónigo y dignidad de tesorero de la catedral de Málaga y deán de la misma desde octubre de 1744.

Promovido con dispensa de grado como arzobispo titular de Trajanapolis in partibus, y ocho días después es nombrado coadministrador de la sede metropolitana de Sevilla, ocupada en esos momentos por el cardenal Luis de Borbón. Consagrado el 16 de marzo de ese mismo año en Madrid por el nuncio de Su Santidad en España monseñor Enríquez.

En 1752 en que fue nombrado obispo de Córdoba y luego arzobispo de Sevilla en 1755 cuyo birrete le impuso el mismo Rey con el cordón de San Genaro ${ }^{5}$. Benedicto XIV lo creó cardenal con el título de los doce apóstoles el 5 de abril de 1756, recibiendo la púrpura y el título de Ss. XII Apostoli el 26 de junio de 1769 tras la elección de Clemente XIV que había ostentado dicho título cardenalicio con anterioridad. Como cardenal no pudo participar en el cónclave de 1758 (Clemente XIII) pero sí lo hará en los de 1769 (Clemente XIV) y 1774 viajando por segunda vez a Roma a la elección del pontífice (Pío VI) ${ }^{6}$.

5 Ballesteros Robles, Luis, Diccionario Biográfico Matritense, Madrid, 1912.

${ }^{6}$ Miranda, Salvador, The Cardinals of the Holy Roman Church: Papal elections of the XVIII century (1700-1775), Florida International University Library (2006). 


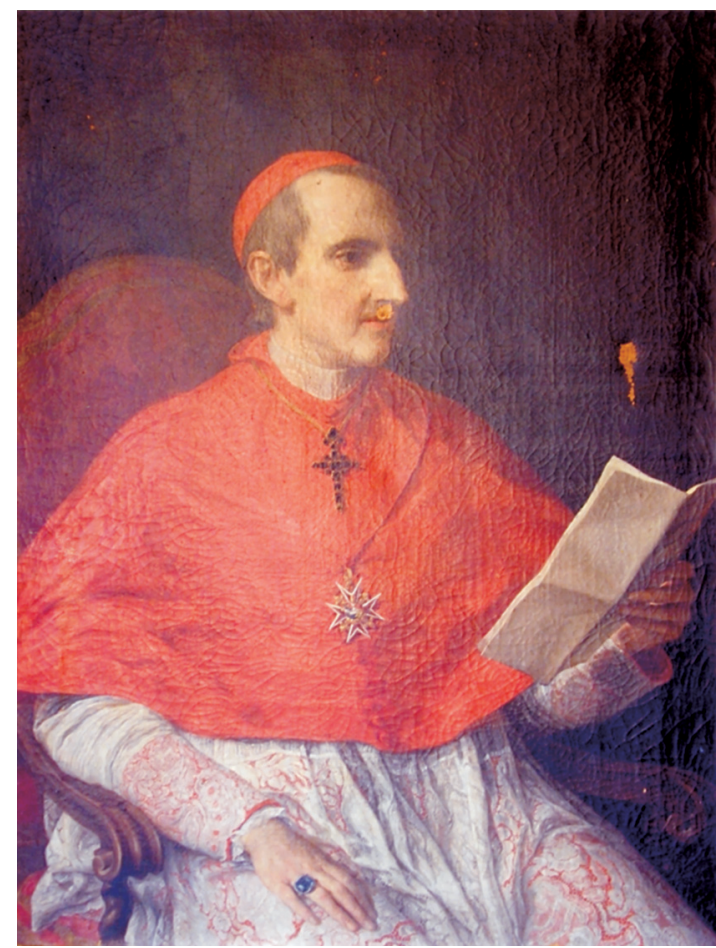

Fig. 3. P. Gerolamo Batoni. Cardenal Solís. Sevilla. Col. Particular.

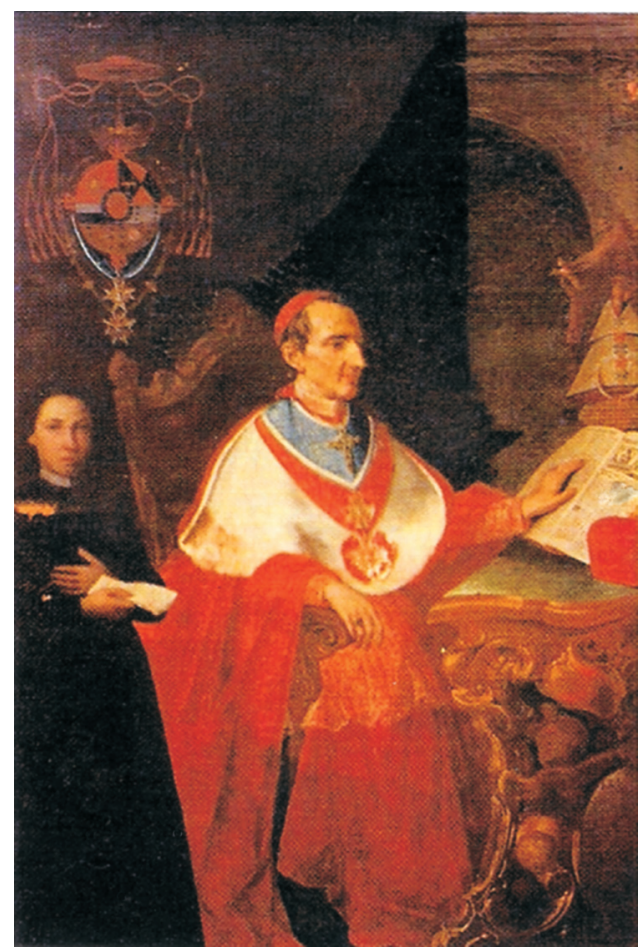

Fig. 4. Cardenal Solís. Convento Santa Rosalía.

Fue también Caballero Gran Cruz de Carlos III. Gran amante del arte e importante patrón en la España de su tiempo, el cardenal Solís aprovechará su estancia en Roma de 1769 para adquirir una serie de obras de arte dentro de las que destacan el retrato realizado por Batoni objeto de este estudio, el magnífico busto en mármol realizado por Juan Adán y el conjunto de esculturas que se instalaron inicialmente en los jardines del palacio arzobispal de Umbrete (Sevilla) y que actualmente se encuentran en Sevilla.

Durante el cónclave de elección de Pío VI (1775) le sorprenderá la enfermedad, falleciendo en Roma el 21 de marzo de 1775, de una pulmonía, a los 62 años de edad. Fue enterrado en la basílica de los Doce Apóstoles y su corazón traído a Sevilla y depositado en el interior del coro alto de la iglesia del convento de Santa Rosalía ${ }^{7}$. El cardenal Solís ejerció su cargo de arzobispo de Sevilla durante el convulso pontificado de Clemente XIV durante el que se disolvió la Compañía de Jesús.

Algunos autores como Carlos Ros subrayan su aspecto de prelado vanidoso y derrochador. Contaba con 75 criados, entre ellos, 5 cocheros y 8 lacayos. Aunque también hay que resaltar su carácter espléndido y generoso con los más necesitados. Las limosnas repartidas por él fueron cuantiosas. Los conventos femeninos resultaron los más favorecidos. Dichas limosnas eran remitidas en dinero, fanegas de trigo y hogazas de pan. En 1764 se vio en la necesidad de pedir al rey una moratoria para sus deudas. En 1764 debía más de 120.000 pesos a varios particulares y en 1764 pidió al cabildo 60.000 pesos para su viaje a Roma ${ }^{8}$.

\footnotetext{
${ }^{7}$ Ros, Carlos, Historia de la Iglesia de Sevilla, Editorial Castillejo, Sevilla, 1992.

8 Ibid.
} 
En la corte romana fue célebre su estancia por el continuo gasto y derroche en funciones religiosas, fiestas y limosnas. Contaba con un gran séquito. Para el cónclave de 1769 gastó 89.113 escudos y 86 bayocos (entre cuyos gastos habría que incluir el de la realización de su retrato por uno de los pintores más cotizados de Europa y sin duda de Roma). Había alquilado un famoso palacio en la Piazza Colonna, invirtiendo grandes sumas en fiestas y recepciones como ya se ha comentado. Se conserva en la biblioteca Colombina una descripción de la magnífica fachada erigida delante de su palacio en honor de la nación Española. Empleó en dicha fachada 13.556 escudos. Grandes fueron sus gastos con motivo de la toma de posesión del título de cardenal presbítero de la basílica de los doce Apóstoles.

Los gastos realizados por el cardenal Solís en las reedificaciones de parroquias y conventos superaron los 4 millones de reales. De su labor como promotor de obras inmobiliarias da buena cuenta el convento de Santa Rosalía y el palacio arzobispal de Umbrete. Aunque la fundación de este convento se hiciera bajo la protección del Arzobispo Jaime de Palafox y Carmona, que anteriormente ocupó la sede de Palermo, de donde trajo la devoción de la Santa palermitana, bajo cuya advocación quiso fundar un convento en Sevilla poniendo al frente como abadesa a su hermana sor Josefa Manuela9 . Se empezó en 1701 la obra y se prolongó hasta 1724, año en que finalizó toda la obra y en el que murió la abadesa. Sin embargo poco iba a durar la obra que con tantos esfuerzos se había realizado, ya que el 13 de agosto de 1761, durante la celebración de una solemne función religiosa, se produjo en la iglesia un devastador incendio que se extendió por todo el convento, destruyéndolo casi por completo. El Cardenal Solís tomo a su cargo la reconstrucción del convento, recabando ayuda de la corona, de particulares y aportando caudales propios ${ }^{10}$. Las obras se ejecutaron rápidamente pues en septiembre de 1762 estaban casi concluidas, trasladándose la comunidad el día 5 de julio del siguiente año con una solemne función religiosa. La dignidad de la reconstrucción se hizo patente especialmente en la Iglesia por especial deseo del cardenal que hubo de justificar el esplendor de la iglesia, poco acorde con la tradicional austeridad del espíritu capuchino, declarando que un templo consagrado a Dios por un príncipe de la Iglesia y por otra parte sometido a su jurisdicción, no podía ser menos que suntuoso ${ }^{11}$. La iglesia está atribuida al arquitecto Diego Antonio Díaz, siendo restaurada tras el incendio por Antonio Matías de Figueroa. El Retablo Mayor del presbiterio es obra del retablista y escultor portugués Cayetano de Acosta, entre 1761 y 1763 , además de otros retablos laterales y con pinturas atribuidas a Juan de Espinal. Aparte de la reconstrucción del edificio hizo varios regalos como un relicario del Lignum Crucis y un crucifijo de porcelana, así como sus retratos que comentaremos más adelante.

El palacio arzobispal de Umbrete sufrió un gravísimo incendio al anochecer el día 27 de febrero de 1762, que tuvo su origen en una chimenea inmediata al dormitorio del cardenal. Poco tiempo después se había empezado a reedificar, y aunque las paredes, especialmente la de su fachada, quedaron ilesas, se derriban para retirarle un poco sobre el jardín, construirlas capaces de sustentar bóvedas y mejorar su planta. Según Justino Matute, el coste de las obras, que se completó replantando la huerta y los jardines, ascendió a 1.500 .000 reales, y añade este autor que el cardenal gastó también en adecentar el hospital de la villa para transeúntes, hombres y mujeres, la cantidad de 40.323 reales.

Las obras del palacio fueron dirigidas por el maestro mayor Ambrosio de Figueroa, durando hasta bien avanzada la década de los años setenta. Tras ello y aprovechando la circunstancia, el cardenal Solís emprendió una tarea mucho más personal, como fue la ampliación y reordenación

\footnotetext{
9 Valdivieso González, E. y Morales Martínez, A., Sevilla oculta. Monasterios y conventos de clausura, Ediciones Guadalquivir, Sevilla, 1980, p. 274.

${ }^{10}$ Ibid.

11 Valdivieso González, E. y Morales Martínez, A., Sevilla oculta. Monasterios y conventos de clausura, Ediciones Guadalquivir, Sevilla, 1980, p. 277.
} 
de los jardines del palacio, influido quizá por lo que había contemplado en Roma en su visita del año 1759, creando en Umbrete lo que no podía hacer en Sevilla por motivos de espacio, un ambiente absolutamente neorrenacentista propio de un príncipe de la Iglesia ${ }^{12}$. Encargó para ello la hechura de una fuente de mármol con la figura infantil del dios Baco, y 26 pedestales de piedra de Morón, estos últimos atribuidos al escultor Cayetano de Acosta, sobre los que se colocaron bustos de personajes mitológicos en mármol y otras figuras en piedras, algunas de ellas parece ser que traídas de Italia, este magnífico conjunto se conserva en parte en el paseo de las Delicias de Sevilla y en varios museos y colecciones particulares, ya que en 1846, cuando el gobierno se incautó del palacio, la fuente y las esculturas fueron trasladadas a Sevilla, donde fueron colocadas en la plaza del Museo, para ser trasladadas en 1864 a su emplazamiento actual.

Aparte se conserva en la catedral de Sevilla una custodia u ostensorio conocido en Sevilla como "el grande" debido a sus proporciones y fastuosidad, o como el ostensorio del cardenal Solís, en honor a su donante, aunque su verdadera advocación es la de San Juan Nepomuceno, cuya imagen figura en el astil, así como los dos episodios más representativos de su vida, que han sido iconografiados en el pie.

Según la inscripción que aparece grabada en la base fue adquirido en Roma el año 1775 por el cardenal poco antes de morir ${ }^{13}$. Sin embargo, dadas las grandes deudas que tenía contraídas y que dejó pendientes, no pudo el cabildo tomar posesión de él hasta que don Ignacio Ceballos, arcediano de Niebla y representante del cabildo para este asunto recibía la orden de entrega de don Manuel Ventura de Figueroa colector general de expolios y vacantes de todo el Reino, si bien advertía a dicho Cabildo "que tuvieran la conbeniente reserba a favor del espolio, por si fallaban los cálculos que se hacían de su caudal y porque quedasen de ese modo prebenidos los perjuicios que en tal caso resultarían a los acreedores". La estructura del ostensorio concuerda con la realizada por la platería italiana durante el tercer cuarto del siglo XVIII y sigue, por tanto, las líneas generales del rococó ${ }^{14}$.

Pompeo Gerolamo Batoni (Lucca 1708-Roma 1787), pintor de gran cultura, precozmente reconocido de una fama internacional. Nacido en Lucca el 25 de enero de 1708 y muerto en Roma el 4 de febrero de 1787. Batoni era el hijo de un conocido orfebre, Paolino Battoni. Se traslada a Roma en 1727 donde realiza su aprendizaje con Sebastiano Conca y Francesco Imperiale (1679-1740). Un aspecto determinante en su formación artística es el estudio de las esculturas de la antigüedad clásica así como de las pinturas de los grandes maestros italianos (Rafael, los Carracci, Correggio,...) de los que realiza numerosos dibujos y copias $^{15}$.

A comienzos de la década de 1740 se independiza y comienza a recibir encargos de manera independiente. En 1743 realiza su celebrada obra "El éxtasis de Santa Catalina de Siena" (Museo de Villa Guinigi, Lucca) donde refleja el refinamiento académico propio de su estilo. En esta década realiza otra de sus obras maestras "La caída de Simón el Mago" (Museo de Arte de Cleveland) inicialmente pintada para la Basílica de San Pedro aunque retirada años más tarde como consecuencia del cambio de tendencia hacia un estilo más simple y menos exuberante. En 1741 es elegido miembro de la Academia de San Lucca en Roma, prueba evidente de su éxito como pintor. A comienzos de la siguiente década ya se ha convertido indiscutiblemente en "el retratista" de Roma, especialmente cuando su máximo rival Antón Raphael Mengs ha partido para España

12 Aguilar Piñal, Francisco, Historia de Sevilla: Siglo XVIII, 3. a ed., Sevilla, Universidad de Sevilla, 1989.

13 Palomero Páramo, Jesús, "La platería en la Catedral de Sevilla", en La Catedral de Sevilla, ediciones Guadalquivir, Sevilla, 1991, p. 27.

${ }^{14}$ Palomero Páramo, Jesús, "La platería en la Catedral de Sevilla", en La Catedral de Sevilla, ediciones Guadalquivir, Sevilla, 1991, p. 27.

15 Belli Barsali, I., Pompeo Batoni. Catalogo della mostra promossa dalla Administrazione Provinciale di Lucca. Lucca, MPF, 1967. 
en 1761. Batoni prefiere a Rafael y a Poussin a los pintores venecianos que se encontraban en boga en aquel momento.

Pompeo Batoni fue un hombre de extensa cultura que obtuvo un enorme prestigio internacional a una edad temprana. Fue el primer artista italiano que desarrolló de forma consciente un estilo artístico alternativo al Rococó y a la pintura veneciana, que pensaba pasados de moda. A través del estudio de Rafael y del primer Renacimiento italiano desarrolló un programa de reforma para la pintura de acuerdo a rigurosas normas académicas ${ }^{16}$. A la par de Mengs, la propuesta de Batoni se transforma en una forma inicial e importante de Neoclasicismo, no como mera y pasiva imitación de la antigüedad sino como una estimulante búsqueda de un ideal de belleza ${ }^{17}$. Es el primer artista italiano que ofrece una interesante alternativa formal al rococó y a la pintura veneciana.

La mano nítida y firme del pintor está bien aplicada también en excelentes retratos, entre los que tendrá una amplia y exquisita clientela entre los que estarán los pontífices, príncipes italianos y por supuesto numerosos comitentes ingleses y alemanes. Con el paso del tiempo sus retratos son cada vez más demandados, que después de 1744 acaparan casi toda su atención ${ }^{18}$. Su estudio es muy frecuentado por extranjeros de paso en la Ciudad Eterna, especialmente británicos que le encargaran sus famosos retratos de cuerpo entero en medio de antigüedades, ruinas y obras de arte. Dichos recuerdos del "Grand Tour" llegaron a proliferar en las colecciones privadas británicas donde se hicieron realmente populares. Sir Joshua Reynolds se convertirá en el principal continuador de este estilo de retrato ${ }^{19}$. En 1769 el doble retrato de José II y Leopoldo II de Austria (Kunsthistorische Museum Viena) le permitió obtener un título nobiliario austriaco ${ }^{20}$. Pompeo Batoni fue también el autor de un retrato del Papa Pío VI (Museos Vaticanos). Precisamente el Papa encargó dicho retrato días después de su elección en el cónclave en el que participó el cardenal Solís pocos días antes de su muerte, que curiosamente guarda gran parecido formal con el retrato que hiciera unos años antes al cardenal, aparece sedente, de tres cuartos y cortado por debajo de las rodillas y también porta en su mano izquierda una carta con la que se identifica el personaje.

Batoni se casó dos veces, con Caterina Setti (muerta en 1742) en 1729, y con Lucia Fattori en 1747 y tuvo doce hijos; de ellos tres fueron colaboradores en su taller. Desde 1759 Batoni vivió en una gran casa en la Vía Bocca di Leone en Roma, que incluía un estudio, salas de exposición y una academia nocturna de dibujo. El artista murió en Roma el 4 de febrero de $1787^{21}$.

El cuadro recientemente descubierto y que como anteriormente mencionamos procede de la colección del marqués de Loreto, se identifica en el siglo XIX en la colección del marqués de Casa León, encontrándose actualmente en una colección particular sevillana, aunque se desconoce por desgracia su mención en inventarios de las colecciones por donde fue pasando, con las mismas dimensiones del conservado en Santa Paula, que es una copia casi idéntica salvo el detalle de la nueva cruz con su correspondiente banda de la orden de Carlos III que se le impuso después de 1769 y por supuesto la firma que aparece en el mismo sitio, o sea al final del reposabrazos del sillón que aquí es la siguiente "Pompeius Battonis pinxit. Rome 1769".

El retratado aparece sedente de media figura en un sillón de estilo Luis XIII, en posición de tres cuartos. Con la mano izquierda sostiene una carta en la que se lee el nombre y dignidad del

16 Clark, Anthony M., Pompeo Batoni. Complete Catalogue, Oxford, Oxford Phaidon, 1985.

17 Zuff, Stefano, La Pittura Italiana, Mondadori edizioni, Milano, 1997.

18 Urrea Fernández, Jesús, La Pintura Italiana del Siglo XVIII en España, Publicaciones del Departamento de Historia del Arte, Valladolid, 1977, p. 244.

19 JACOB, John, Pompeo Batoni (1708-1787) and his british patrons, London, Greater London Council, 1982.

20 Belli Barsali, I., Pompeo Batoni. Catalogo della mostra promossa dalla Administrazione Provinciale di Lucca, Lucca, MPF, 1967.

${ }^{21}$ Borelli, E., Pompeo Batoni (Lucca 1708-1787), Lucca, Pacini Facci, 1967. 
retratado "Al em. ${ }^{\circ}$ Ecmo S. Card de Solís Arz. de Sevilla B.L P ss." (cosa común de los retratos que hace Battoni para identificar a los personajes). La cabeza, cubierta con el habitual solideo episcopal, está girada casi de perfil, cosa habitual de los retratos del cardenal por su condición de bizco, y el magro rostro con la prominente nariz de tipo aguileño deja vislumbrar una interesante personalidad. Está vestido con un fino roquete de encaje en donde se observa la hábil calidad del pintor, encima del cual lleva una esclavina púrpura sobre la que cuelga su cruz episcopal y la banda y condecoración de San Genaro, la otra mano, o sea la izquierda reposa apaciblemente extendida en el regazo luciendo en el dedo anular el majestuoso anillo episcopal.

En cambio la copia del palacio arzobispal, que según algunos catálogos se considera anónima, aunque muy segura es la hipótesis que sea también de Rubira, presenta algunas variantes como sería el recorte que se hace del retratado siendo ahora casi un busto y por tanto eliminándose las manos y el regazo, incluyendo sin embargo el escudo episcopal en el ángulo superior derecho y una leyenda que ocupa toda una franja inferior en la que se lee "El em. Y exc. D: Franc. ${ }^{\circ}$ de Solis Folch de Cardona. Presb. Cardenal de la SRY del titulo de la basílica de los doce apóstoles, caballero gran cruz de la real y distinguida orden de Carlos III de la real orden de San Genaro y del de Calatrava. Obispo que fue de Córdoba y Arzobispo de Sevilla del Cons. De SM." Y otra leyenda en la zona superior que indica el año y a la edad en que murió.

Otra versión de estas copias sería la existente en el convento de Santa Rosalía (que él tanto beneficiara) que desconocemos su autor, en donde aparece el cardenal sedente, ahora de cuerpo entero acompañado de un criado o monaguillo que observa al espectador y porta una carta en la que desvela la identidad del personaje, mientras la otra la mantiene en solemne actitud en el pecho. El cardenal ahora, en vez de portar la carta, posa la mano izquierda sobre un libro abierto, al lado del cual hay una pequeña imagen mariana aludiendo a la devoción a la Virgen por parte del cardenal, que descansa en una bella consola barroca. También se ha introducido alguna novedad como el cortinaje del fondo que deja entrever parte de un arco de medio punto como un toque de efecto teatral delante del cual aparece el escudo episcopal ${ }^{22}$.

El otro gran cliente de Arte, la Iglesia, no tiene en este siglo prácticamente ningún interés por la pintura italiana, salvo raras excepciones como el conjunto de pinturas para la clerecía de Salamanca. Ponz, buen conocedor de lo italiano del siglo, sólo hace mención de alguna obra que se importa desde Italia (sin contar, claro, los artistas oriundos de Italia que trabajan en España como los artistas genoveses que hay en Cádiz). El cardenal Borja retratado por Procaccini, el cardenal Solís retratado por Batoni y más tarde el cardenal Despuig, retratado por Tofanelli, junto con el chantre de la catedral de Teruel don Joaquín García constituyen ejemplos aislados del gusto refinado y distinguido dentro el clero aristocrático español ${ }^{23}$.

Jesús Porres Benavides y MANuel SÁNChez LóPIZ

\footnotetext{
${ }^{22}$ También reseñar que en la Capilla del Bautismo de la parroquial de Chipiona (Cádiz) existe otra versión del realizado por Battoni, de buena factura y algo mayor de proporciones.

${ }^{23}$ Urrea Fernández, Jesús, La Pintura Italiana del Siglo XVIII en España, Publicaciones del Departamento de Historia del Arte, Valladolid, 1977, p. 53.
} 